\title{
BMJ Open Risk of infection associated with intravenous iron preparations: protocol for updating a systematic review
}

Akshay Shah, ${ }^{1}$ Anita Sugavanam, ${ }^{2}$ Jack Reid, ${ }^{2}$ Antony J Palmer, ${ }^{3}$ Edward Dickson, ${ }^{4}$ Susan Brunskill, ${ }^{5}$ Carolyn Doree, ${ }^{6}$ Charles M Oliver, ${ }^{\circ} 7$ Austin Acheson, ${ }^{8}$ Ravi Rao Baikady, ${ }^{9}$ Sohail Bampoe, ${ }^{10}$ Edward Litton, ${ }^{11}$ Simon Stanworth ${ }^{12}$

To cite: Shah A, Sugavanam A,

Reid J, et al. Risk of infection associated with intravenous iron preparations: protocol for updating a systematic review. BMJ Open 2019;9:e024618. doi:10.1136/ bmjopen-2018-024618

- Prepublication history andadditional material for thispaper are available online. Toview these files, please visitthe journal online (http://dx. doi.org/10.1136/bmjopen-2018024618)

Received 5 June 2018 Revised 11 February 2019 Accepted 20 May 2019
Check for updates

(C) Author(s) (or their employer(s)) 2019. Re-use permitted under CC BY-NC. No commercial re-use. See rights and permissions. Published by BMJ.

For numbered affiliations see end of article.

Correspondence to

Dr Akshay Shah;

akshay.shah@ndcls.ox.ac.uk

\section{ABSTRACT}

Introduction The benefits and risk of intravenous iron have been documented in previous systematic reviews and continue to be the subject of randomised controlled trials (RCTs). An ongoing issue that continues to be raised is the relationship between administering iron and developing infection. This is supported by biological plausibility from animal models. We propose an update of a previously published systematic review and meta-analysis with the primary focus being infection.

Methods and analysis We will include RCTs and non-randomised studies (NRS) in this review update. We will search the relevant electronic databases. Two reviewers will independently extract data. Risk of bias for RCTs and NRS will be assessed using the relevant tools recommended by The Cochrane Collaboration. Data extracted from RCTs and NRS will be analysed and reported separately. Pooled data from RCTs will be analysed using a random effects model. We will also conduct subgroup analyses to identify any patient populations that may be at increased risk of developing infection. We will provide a narrative synthesis on the definitions, sources and responsible pathogens for infection in the included studies. Overall quality of evidence on the safety outcomes of mortality and infection will be assessed using the Grading of Recommendations, Assessment, Development and Evaluation approach. Ethics and dissemination This systematic review will only investigate published studies and therefore ethical approval is not required. The results will be broadly distributed through conference presentations and peerreviewed publications.

Trial registration number PROSPERO

(CRD42018096023).

\section{INTRODUCTION}

Treating anaemia is a key pillar of patient blood management and a recent James Lind prioritisation exercise ranked the timely identification of anaemia and treatment as a top 10 priority for research into blood transfusion and blood donation. ${ }^{1}$ Systematic reviews have shown the efficacy of intravenous iron with regards to treating anaemia and reducing
Strengths and limitations of this study

- Systematic review protocol primarily focusing on a safety outcome (risk of infection) with intravenous preparations.

- Comprehensive review that will include data from randomised controlled trials and non-randomised studies.

- Infection is not often a predefined endpoint in published studies and definitions of infection will vary across studies.

- There will be considerable heterogeneity in participant populations, doses and types of intravenous iron used and follow-up time points.

blood transfusion requirements, ${ }^{2}{ }^{3}$ although with varying degrees of effect size and the primary outcomes in majority of trials were haematological (change in haemoglobin concentration, transfusion requirements) instead of clinical outcomes (eg, quality of life).

Despite the widespread use of intravenous iron, ${ }^{12}$ uncertainty persists as to whether intravenous iron is associated with an increased risk of infection. The uncertain relationship between iron and infection has long been postulated and remains a topic of interest in ongoing trials of oral iron, for example, in the setting of malaria and other tropical infections in low-resource country settings. ${ }^{4}$ Iron is essential for extracellular pathogens as it an ideal redox catalyst for important cellular processes such as respiration and DNA replication. ${ }^{5}$ Humans are able to withhold free (non-transferrin-bound) iron from invading pathogens through a process termed nutritional immunity in an effort to limit infection. ${ }^{56}$ Intravenous iron administration can lead to increased levels of circulating free iron, which can be detrimental to the host and promote pathogen growth. Such 
an interaction is supported by biological plausibility in recent animal models where the administration of intravenous iron worsening shock, lung injury and mortality. ${ }^{7}$

Two recent systematic reviews have investigated the efficacy and safety of intravenous iron therapy. ${ }^{27}$ The first systematic review identified 72 randomised controlled trials (RCTs) that included 10605 participants. The authors reported a reduced risk of requirement for red blood cell (RBC) transfusion (risk ratio (RR) 0.74, $95 \%$ CI 0.62 to $0.88 ; 22$ RCTs, 3321 participants). Of note, this potential benefit was counterbalanced a significantly increased risk of infection (RR 1.34, 95\% CI 1.10 to 1.64; 24 RCTs, 4400 participants) when intravenous iron was compared with oral iron or no iron. ${ }^{2}$ The second systematic review pooled data from 32 RCTs and showed a point estimate which again favoured infection, although this was statistically non-significant (RR $1.17 ; 95 \%$ CI 0.83 to 1.65$).{ }^{8}$ Interpreting data on infection from these meta-analyses is challenging because infection is not always defined as a prespecified, standardised outcome measure in RCTs but rather reported as safety outcome. A recent editorial highlighted the need for an adequately powered trial of intravenous iron with infection as a primary outcome. ${ }^{6}$

Given the ongoing uncertainty regarding the risk of infection, the primary objective of this systematic review was to update the previous review by Litton $e t a \hat{l}$ by identifying and incorporating recent trial data to evaluate the safety data for intravenous iron on the risk of infection across all clinical settings. A better understanding of the characterisation of infection in patients receiving iron therapy will help inform the design of subsequent trials in particular groups of patients (eg, critically ill, emergency surgery) in whom the risk of infection is of clinical concern. Our secondary objective is to continue to collect efficacy data to focusing primarily on changes in haemoglobin concentration, transfusion requirements and functional outcomes.

\section{METHODS}

We used the Preferred Reporting Items for Systematic Review and Meta-Analysis (PRISMA) Protocols reporting guidelines. ${ }^{9}$ Studies will be selected according to the criteria outlined below.

\section{Eligibility criteria}

We will include RCTs from 1 January 2013 onwards as the last search date for the previous review was June $2013 .{ }^{2} \mathrm{We}$ will also include non-randomised studies (NRS) in this updated review as infection may not always be reported in RCTs and the findings of infection outcomes reported in NRS may be useful to inform the design of a future RCT. We will only include NRS that meet the following criteria:

- Published since 1 January 2007 as this is the year from which newer intravenous iron preparations (Ferinject, Monofer, Venofer, Injectofer) received and/or renewed their marketing authorisation. Therefore, any data extracted is likely to be reflective of current practice. Studies evaluating low molecular weight dextran (INFed, Cosmofer), ferumoxytol, ferric pyrophosphate citrate (TriFeric) and iron polymaltose will also be included.

- At least two comparable groups (including controlled before and after, and prospective/retrospective cohort studies).

- Quasi-RCTs.

- Provide data on our primary outcome of infection.

We will exclude any studies that provide no outcome data of interest, NRS published before 1 January 2007 and NRS that do not have an intravenous iron comparison arm. We will include studies examining all participant populations (including paediatrics, pregnancy) but excluding healthy volunteers. Included studies would compare intravenous iron to no iron/placebo or oral iron.

Our primary outcome of interest is the number of patients who develop an infection as defined by the study authors. Secondary safety and efficacy outcomes include:

- Mortality—short-term ( $\leq 30$ days), long-term (>30 days).

- Hospital length of stay.

- Change in haemoglobin concentration from baseline/pretreatment levels to end of study period.

- Transfusion requirements during study period (\% transfused, mean number of RBC units transfused).

\section{Information sources and search strategy}

We will search the following databases for RCTs (from 1 January 2013), systematic reviews and NRS (from 1 January 2007)-Cochrane Central Register for Controlled Trials; Medline (Ovid interface); Ovid Interface; Cumulative Index to Nursing and Allied Health Literature (CINAHL); Transfusion Evidence Library; Web of Science Conference Proceedings Citation Index Science. This will be supplemented by searching ongoing trial databases such as ClinicalTrials.gov and WHO International Clinical Trials Search Registry Platform. Citation lists of included studies and relevant reviews will also be scanned to identify any studies missed by the search. A draft Medline search strategy is included in online supplementary appendix 1 .

\section{Study selection}

Literature search results will be uploaded to Covidence, a web-based software platform, to facilitate citation screening between reviewers. Review authors will independently screen the titles and abstracts yielded by the search against the prespecified inclusion criteria. Two review authors will then independently screen the fulltext reports and decide whether these meet the inclusion criteria. Disagreements will be resolved through discussion, and if necessary, referred to a third reviewer. The study selection process will be reported in a PRISMA flow diagram. 


\section{Data extraction}

For RCTs, two reviewers will use the data extraction form used for the original review to extract data independently. We will standardise and pilot a data extraction form for NRS and items for extraction from NRS will include:

- Data on confounding factors.

- Comparability of groups based on the consideration of confounding factors.

- Methods used to control for confounding.

- Effect estimates-both adjusted and unadjusted if available.

For both sets of studies, we will extract the following additional data, if reported, on the outcome of infection:

- Definition of infection used (ie, guideline based, laboratory based, clinical discretion).

- Site of infection (eg, lung, wound, gastrointestinal).

- Reporting of identified pathogens.

- Antibiotic usage.

Disagreements will be resolved through discussion, and if necessary, referred to a third reviewer. We will contact study authors to resolve any uncertainties.

\section{Risk-of-bias assessment}

Risk of bias for the RCTs will be reported using the Cochrane Risk of Bias tool. ${ }^{10}$ For the non-RCT data, risk of bias will be reported using the Risk of Bias in Non-Randomized Studies - of Interventions (ROBINS-I) developed by the Cochrane Bias Methods Group. ${ }^{11}$ Two reviewers will make these judgements independently.

\section{Data synthesis}

Data from RCTs and NRS will be analysed and reported separately.

\section{RCTs}

The primary endpoint will be the proportion of participants who developed an infection. Dichotomous outcomes (infection, mortality, requirement for blood transfusion) will be reported as RRs with corresponding 95\% CIs. Continuous outcomes will be reported as weighted mean (with $95 \% \mathrm{CI}$ ) or standardised mean differences $(95 \%$ CI) as appropriate. For continuous measures, the mean difference in change from baseline values between groups will be used preferentially; if change from baseline values is not reported, then the mean difference in measures at follow-up will be used. The unit of analysis will be per individual randomised. Data from included studies will be pooled for meta-analysis using a random effects model. Statistical heterogeneity will be tested using the $\mathrm{I}^{2}$ statistic and $\mathrm{I}^{2}>50 \%$ will be considered as substantial heterogeneity. If substantial heterogeneity is present among the trials, the study characteristics of the included studies will be analysed and we will attempt to explain the heterogeneity by subgroup analysis or sensitivity analysis. If sufficient data are available, we will undertake metaregression to examine the effect of cumulative intravenous iron dose and the incidence of infection in the control group (as a surrogate for background/endemic burden of infection) on our primary outcome. Statistical analysis will be conducted on RevMan V.5.1. and STATA (V.14, StataCorp LP, College Station, Texas, USA).

\section{NRS}

For NRS, we will only report results descriptively on the primary outcome of infection instead of pooling results due to heterogeneity in clinical conditions, study designs and variations in statistical adjustment. If possible, results will be displayed in a forest plot, with studies sorted according to study design features, and the pooled estimate will be suppressed as recommended by the Cochrane Collaboration. ${ }^{6}$

\section{Analysis of subgroups}

Subgroup analysis of the primary safety outcome (infection) will be performed on the following:

- Clinical settings (in-patient medical (any), outpatient (any), elective surgical, non-elective (urgent/emergency) surgical, obstetrics, paediatrics, critically ill).

- Different iron profiles at enrolment as defined by the study authors (true iron deficiency, functional iron deficiency, iron-restricted erythropoiesis).

- Mode of administration (eg, single dose, continuous infusion, multiple boluses).

- Cumulative dose of intravenous iron.

- Incidence of infection in the control group.

We will carry out a sensitivity analysis on infection and mortality outcomes by excluding studies with a high risk of bias. We will assess for publication bias on the primary outcome with a funnel plot if $\geq 10$ studies are available, plotting the OR for proportion that develop infection against the SE of the log OR.

A systematic narrative synthesis will be provided with information presented in the text and tables to summarise data on infection provided in the include studies. This narrative synthesis will explore the definitions of infection used, reporting of infection source and pathogens and antibiotic use.

\section{Confidence in cumulative evidence}

According to the Grading of Recommendations, Assessment, Development and Evaluation (GRADE) approach, we will assess the overall quality of evidence for the main safety outcomes of infection and mortality. ${ }^{12}$ In line with current GRADE guidance, if the certainty of evidence differs between RCTs and NRS, we will present summary of findings tables for the higher certainty of evidence. If the certainty ratings are the same, results from both bodies will be presented separately. ${ }^{13}$

\section{Patient and public involvement}

Patients and members of the public were not directly involved in the design of this study. However, appropriate management of anaemia, through interventions such as iron therapy, has been identified as a key research priority in a recent James Lind Priority Setting Partnership exercise. ${ }^{1}$ 


\section{DISCUSSION}

Recent patient blood management efforts have attempted to reduce blood transfusion by using alternative therapies such as intravenous iron. Safety concerns surrounding older preparations, mainly anaphylaxis, have been allayed by the development of newer, stable preparations which has led to intravenous iron being used more frequently in multiple settings. ${ }^{14}$ Despite its widespread use, concerns surrounding infection remain both from systematic reviews and animal models.

Our review will provide an up to date and comprehensive estimate of the risk of infection associated with intravenous iron preparations across multiple patient groups. In addition, we will also provide data on the characterisation of infection as a step towards standardising infection as an outcome measure for future trials of intravenous iron.

\section{Author affiliations \\ ${ }^{1}$ Radcliffe Department of Medicine, University of Oxford, Oxford, UK \\ ${ }^{2}$ Department of Anaesthetics, Brighton and Sussex University Hospitals NHS Trust, \\ Brighton and Hove, UK \\ ${ }^{3}$ Nuffield Department of Orthopaedics, Rheumatology and Musculoskeletal \\ Sciences, University of Oxford, Oxford, UK \\ ${ }^{4}$ Department of Colorectal Surgery, Oxford University Hospitals NHS Foundation \\ Trust, Oxford, UK \\ ${ }^{5}$ Systematic Review Initiative, NHS Blood and Transplant, Oxford, UK \\ ${ }^{6}$ NHS Blood and Transplant, John Radcliffe Hospital, Oxford, UK \\ ${ }^{7}$ Division of Biosciences, University College London, London, UK \\ ${ }^{8}$ Department of Colorectal Surgery and NIHR Biomedical Research Unit in \\ Gastrointestinal and Liver Diseases, Queen's Medical Centre, Nottingham, UK \\ ${ }^{9}$ Department of Anaesthetics, Perioperative Medicine and Pain, Royal Marsden NHS Foundation Trust, London, UK \\ ${ }^{10}$ Department of Anaesthesia and Perioperative Medicine, University College London Hospitals NHS Foundation Trust, London, UK \\ ${ }^{11}$ Intensive Care Unit, St John of God Hospital, Perth, Western Australia, Australia \\ ${ }^{12}$ Department of Haematology/Transfusion Medicine, Oxford Radcliffe Hospitals Trust, Oxford, UK}

Contributors AS, AS, SS and EL conceived the idea for the review update. AS prepared the initial protocol draft with input from SB and SS who contributed to the revision of the manuscript. CD will perform the search and AS, JR, AJP and ED will screen and extract data. All listed authors, including CMO, AA, RRB and $\mathrm{SB}$, contributed to the development of the idea and drafting and revisions of the manuscript.

Funding AS is funded by an NIHR Doctoral Research Fellowship (DRF-2017-10094). This research received no specific grant from any funding agency in the public, commercial or not-forprofit sectors.
Competing interests RRB has received educational grants/honorarium from Pharmacosmos UK and Vifor Pharma. AA's research department has received grant support from Syner-Med, UK, Pharmacosmos, Denmark and Vifor Pharma, Switzerland. Honoraria or travel support received for lecturing from the following companies: Ethicon Endosurgery; Johnson and Johnson, UK; Olympus, Essex, UK; Vifor Pharma, Glattbrugg, Switzerland and Pharmacosmos, Denmark.

Patient consent for publication Not required.

Provenance and peer review Not commissioned; externally peer reviewed.

Open access This is an open access article distributed in accordance with the Creative Commons Attribution Non Commercial (CC BY-NC 4.0) license, which permits others to distribute, remix, adapt, build upon this work non-commercially, and license their derivative works on different terms, provided the original work is properly cited, appropriate credit is given, any changes made indicated, and the use is non-commercial. See: http://creativecommons.org/licenses/by-nc/4.0/.

\section{REFERENCES}

1. Hibbs SP, Brunskill SJ, Donald GC, et al. Setting priorities for research in blood donation and transfusion: outcome of the James Lind Alliance priority-setting partnership. Transfusion 2019;59.

2. Clevenger B, Gurusamy K, Klein AA, et al. Systematic review and meta-analysis of iron therapy in anaemic adults without chronic kidney disease: updated and abridged Cochrane review. Eur J Heart Fail 2016;18:774-85.

3. Litton E, Xiao J, Ho KM, Km H. Safety and efficacy of intravenous iron therapy in reducing requirement for allogeneic blood transfusion: systematic review and meta-analysis of randomised clinical trials. BMJ 2013;347:f4822.

4. Pasricha SR, Armitage AE, Prentice AM, et al. Reducing anaemia in low income countries: control of infection is essential. BMJ 2018;362:k3165

5. Cassat JE, Skaar EP. Iron in infection and immunity. Cell Host Microbe 2013;13:509-19.

6. Youssef LA, Spitalnik SL. Iron: a double-edged sword. Transfusion 2017;57:2293-7.

7. Suffredini DA, Xu W, Sun J, et al. Parenteral irons versus transfused red blood cells for treatment of anemia during canine experimental bacterial pneumonia. Transfusion 2017;57:2338-47.

8. Avni T, Bieber A, Grossman A, et al. The safety of intravenous iron preparations: systematic review and meta-analysis. Mayo Clin Proc 2015;90:12-23.

9. Moher D, Shamseer L, Clarke M, et al. Preferred reporting items for systematic review and meta-analysis protocols (PRISMA-P) 2015 statement. Syst Rev 2015;4:1.

10. Higgins JP, Altman DG, Gøtzsche PC, et al. The Cochrane Collaboration's tool for assessing risk of bias in randomised trials. BMJ 2011;343:d5928.

11. Sterne JA, Hernán MA, Reeves BC, et al. ROBINS-I: a tool for assessing risk of bias in non-randomised studies of interventions. BMJ 2016;355:i4919.

12. Atkins D, Best D, Briss PA, et al. Grading quality of evidence and strength of recommendations. BMJ 2004;328:1490.

13. Schünemann HJ, Cuello C, AkI EA, et al. GRADE guidelines: 18. How ROBINS-I and other tools to assess risk of bias in nonrandomized studies should be used to rate the certainty of a body of evidence. $J$ Clin Epidemiol 2018.

14. Auerbach M, Macdougall IC. Safety of intravenous iron formulations: facts and folklore. Blood Transfus 2014;12:296-300. 